\title{
DESIGN AND ANALYSIS OF X-Y- $\theta$ POSITIONING STAGE BASED ON REDUNDANT PARALLEL LINKAGE
}

\author{
U.Sudhakar $^{1}$, J.Srinivas ${ }^{2}$ \\ ${ }^{1}$ Assistant Professor, Department of Mechanical Engg., GMRIT, Rajam, mr.u.sudhakar@gmail.com \\ ${ }^{2}$ Associate Professor, Department of Mechanical Engg., NIT Rourkela, 769008, Odisha, srin07@yahoo.co.in
}

\begin{abstract}
This paper presents the concept of a planar positioning stage based on a kinematically redundant parallel linkage. Basic kinematics and workspace analysis of base redundant manipulator is initially explained and the procedure of static analysis to predict the actuated joint torques is described. As there are six actuators in the linkage, the redundancy can be overcome by proper selection of the base joint variables. Also it is assumed that the motion is at a constant speed. A numerical example is shown with a straight line trajectory to illustrate the workspace and joint force calculation aspects of this linkage. The possible arrangement of the stage with electrostatic actuation and sensing are finally highlighted.
\end{abstract}

Keywords: Kinematic redundancy, Parallel mechanism, Static analysis, and Workspace characteristics.

\section{INTRODUCTION}

In precision engineering, the positioning systems delivering high strokes with compact sizes are essentially required. Most of the positioning stages use parallel kinematic mechanisms, which have a fixed base and movable end-effector connected by multiple (at least two) independent kinematic chains in a parallel scheme. The number of independent kinematic chains is equal to the number of DOF of the end-effector. Spatial positioning devices are often based on hexapods or tripods. A typical example is the 6-DOF Stewart platform [1], in which the platform is supported and driven by six struts (prismatic joints). Another successful application is the Delta robot [2] where a parallelogram is used to allow an output link to move without changing its orientation with respect to an input link. The three parallelograms completely prevent the platform from rotating and make the Delta robot an XYZ stage. Other examples include a tetrahedral tripod [3], which is a threecylindrical-prismatic-revolute XYZ translation stage. Three prismatic joints are used to drive the platform by varying strut lengths. This design is simple, singularity-free and efficient. It provides a stiff and compact platform for machine tool construction with high workspace-to-machine-volume ratio. When it comes to the three degrees of freedom positioning, commercially called $\mathrm{x}$-y-theta positioning tables based on serial linkages are used. In serial linkages, the first actuator has to support the weight of other actuators resulting in a large and sluggish device. For the last one decade, planar parallel linkages have received considerable attention as positioning devices.

Main disadvantage of parallel kinematic linkages is that endeffector can only move within the intersection of the workspaces of the kinematic chains. This can limit the workspace of the stage. A direct result of the small workspace is the poor dexterity and being constrained to the intersection of the workspaces of several kinematic chains. The workspace of a parallel manipulator generally contains loci of parallel or type-2 singularities. In these singular configurations, the manipulator cannot sustain certain wrench applied to its endeffector. Redundancy is one of the means to reduce or eliminate some of the limitations of parallel manipulators. In a statically determinate system, equilibrium equations can be used to determine the reaction forces at the constraints when external forces are applied. Redundant parallel actuation can also help-in improving damping, preload a structure in addition to prevent the singular positions. There are two types of redundancies in practice. First one is actuation redundancy, which consists of either actuating normally passive joint or adding additional actuated branches in an existing manipulator. For sustaining a given wrench by the endeffector, there are an infinite number of possible solutions for the actuator torques. On the other hand, kinematic redundancy consists of adding extra links and actuators. In this case, the mobility of a manipulator is greater than the number of degrees-of-freedom and there are an infinite number of possible solutions for the inverse kinematic problem.

Several researchers illustrated the effectiveness of redundancy in parallel kinematic linkages. Wang and Gosselin [4] introduced one degree of kinematic redundancy in each leg of a planar manipulator, a spherical manipulator and a spatial manipulator. It was shown that the singularity configurations were significantly reduced when compared to the nonredundant manipulator. Mohamed and Gosselin [5] used kinematic redundancy to reconfigure the platform of the 
parallel manipulators. Ebrahimi et al. [6] analyzed a 3-PRRR manipulator and showed that kinematic redundancy enlarges the workspace and can completely eliminate singularities within it. Kotlarsky et al. [7] added one prismatic actuated joint in one leg of a 3-RRR manipulator and used different optimization strategies to avoid singularities and minimize the pose errors. More recently, Boudreau and Nokleby [8] presented a redundant parallel manipulator configuration for resolving generalized forces during tracking of a trajectory. Application of a parallel redundant configuration in a motion system with the aim of minimizing the storage of elastic energy in structure during acceleration is of recent concern. More recent works aim at design of positioning stages using mechanism redundancy. Ahn et al. [9] presented a micro parallel positioning platform with two translational motions and a rotational motion and employed it for simulation of cutter path in laser-micromachining. Krishnan and Saggere [10] proposed a compliant parallel redundant linkage for micromanipulation tasks.

\subsection{Objectives of Present Work}

Based on the available literature, it is found that very few works employed redundant parallel linkages in positioning stages. In spite of their advantages, there may be disadvantages like mechanical imperfection and friction due to additional links and joints in a redundant linkage. Objectives of present work are (i) illustrate the kinematics of 3-PRPR linkage. (ii) Prediction of workspace and singularity characteristics (iii) Perform static analysis during path tracking application and (iv) Propose the stage for micro scale application. Organization of the present paper is as follows: Section-2 describes the kinematic analysis and workspace determination. Section-3 deals with torque estimation methodology. Results and discussions are presented in section-4.

\section{DESCRIPTION OF POSITIONING STAGE}

A recently proposed 3-PRPR manipulator gives interesting insights. Fig. 1 shows this linkage. An actuated prismatic joint fixed to the base (base prismatic joint) is added. It is followed by a passive revolute joint, an actuated prismatic joint (distal prismatic joint) and a passive revolute joint attached to the mobile platform. The points $\mathrm{O} 1, \mathrm{O} 2$ and $\mathrm{O} 3$ and $\mathrm{B} 1, \mathrm{~B} 2$ and $\mathrm{B} 3$ are forming two equilateral triangles. The fixed reference frame and the mobile frame attached to the end-effector are at the geometric centers of triangles $\mathrm{O} 1 \mathrm{O} 2 \mathrm{O} 3$ and $\mathrm{B} 1 \mathrm{~B} 2 \mathrm{~B} 3$ with origins at $\mathrm{O}$ and $\mathrm{P}$, respectively.

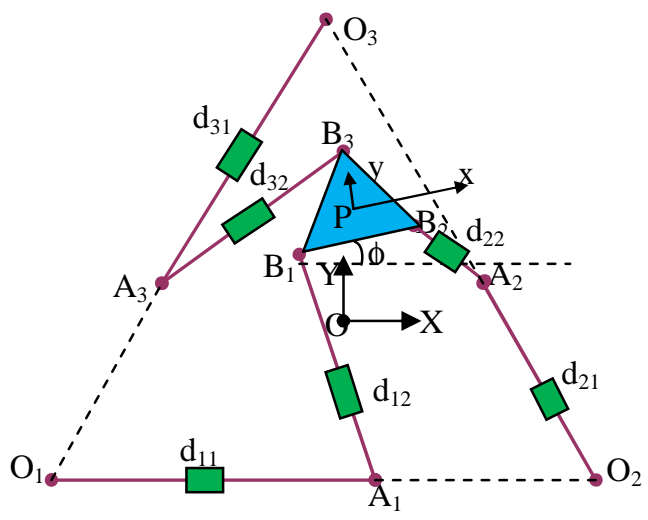

Fig.1 Redundant 3-PRPR planar parallel platform

There are now six actuators and the length of the $\mathrm{j}^{\text {th }}$ actuator $(j=1,2)$ of branch $i(i=1,2,3)$ is represented by $d_{i j}$. The coordinates of points $\mathrm{B}_{\mathrm{i}}$ referring to fixed frame $\mathrm{XOY}$ is:

$$
\left\{\mathrm{OB}_{\mathrm{i}}\right\}=\{\mathrm{OP}\}+[\mathrm{R}]\left\{\mathrm{PB}_{\mathrm{i}}^{\prime}\right\}
$$

Where $[R]$ is the rotation matrix $\left[\begin{array}{cc}\cos \phi & -\sin \phi \\ \sin \phi & \cos \phi\end{array}\right]$, vector $\mathrm{OP}=\left\{\begin{array}{l}\mathrm{x} \\ \mathrm{y}\end{array}\right\}$ and $\left\{\mathrm{PB}_{\mathrm{i}}^{\prime}\right\}$ is vector from $\mathrm{P}$ to $\mathrm{B}_{\mathrm{i}}$ expressed in moving frame $\mathrm{x}-\mathrm{P}-\mathrm{y}$.

Thus,

$$
\begin{aligned}
& \mathrm{x}_{\mathrm{Bi}}=\mathrm{x}+\left(\mathrm{PB}^{\prime}{ }_{\mathrm{ix}} \cos \phi-\mathrm{PB}^{\prime}{ }_{\mathrm{iy}} \sin \phi\right) \\
& \mathrm{y}_{\mathrm{Bi}}=\mathrm{y}+\left(\mathrm{PB}^{\prime}{ }_{\mathrm{ix}} \sin \phi-\mathrm{PB}_{\mathrm{iy}}^{\prime} \cos \phi\right)
\end{aligned}
$$

Also, the length of each actuator is given by:

$$
\mathrm{d}_{\mathrm{i} 2}{ }^{2}=\left(\mathrm{x}_{\mathrm{Bi}}-\mathrm{x}_{\mathrm{Ai}}\right)^{2}+\left(\mathrm{y}_{\mathrm{Bi}}-\mathrm{y}_{\mathrm{Ai}}\right)^{2}
$$

Here, $\left(\mathrm{x}_{\mathrm{Ai}}, \mathrm{y}_{\mathrm{Ai}}\right)$ are the coordinates of the points $\mathrm{A}_{\mathrm{i}}$ referred to fixed coordinate frame XOY, which are nothing but the displacements $d_{i 1}$ of the extra actuators.

\subsection{Workspace}

The so-called constant-orientation workspace is the set of positions attainable by the platform center for a fixed orientation of the platform, given the actuator limits as inputs. Referring to Fig.1, the workspace circles are given by:

$$
\begin{aligned}
& \left\{x-r \cos (\phi+\pi / 6)-d_{11}\right\}^{2}+\{y-r \sin (\phi+\pi / 6)\}^{2}=d_{12}^{2} \\
& \left\{x-r \cos (\phi+\pi / 6)+\ell \cos \phi-\left(t_{1}-d_{21} \cos (\pi / 3)\right\}^{2}\right. \\
& \quad+\left\{y-r \sin (\phi+\pi / 6)+\ell \sin \phi-d_{21} \sin (\pi / 3)\right\}^{2}=d_{22}^{2}
\end{aligned}
$$




$$
\begin{aligned}
& \left\{x-r \cos (\phi+\pi / 6)+\ell \cos (\phi+\pi / 3)-\left(\mathrm{t}_{2}-\mathrm{d}_{31} \cos (\pi / 3)\right\}^{2}\right. \\
& \quad+\left\{\begin{array}{l}
\mathrm{y}-\mathrm{r} \sin (\phi+\pi / 6)+\ell \sin (\phi+\pi / 3) \\
-\left(\mathrm{t}_{3}-\mathrm{d}_{31} \sin (\pi / 3)\right.
\end{array}\right\}^{2}=\mathrm{d}_{32}^{2}
\end{aligned}
$$

\subsection{Jacobian Analysis}

A Jacobian matrix is necessary in order to derive the dynamic equation of manipulator. The Jacobian matrix is the relation of small perturbation of input and the response of output. In order to find Jacobian matrix, time-derivative of Eq.(3) yields:

$$
2 \mathrm{~d}_{\mathrm{i} 2} \dot{\mathrm{d}}_{\mathrm{i} 2}=2\left(\dot{\mathrm{x}}_{\mathrm{Bi}}-\dot{\mathrm{x}}_{\mathrm{Ai}}\right)\left(\mathrm{x}_{\mathrm{Bi}}-\mathrm{x}_{\mathrm{Ai}}\right)+2\left(\dot{\mathrm{y}}_{\mathrm{Bi}}-\dot{\mathrm{y}}_{\mathrm{Ai}}\right)\left(\mathrm{y}_{\mathrm{Bi}}-\mathrm{y}_{\mathrm{Ai}}\right)
$$

Here,

$$
\begin{gathered}
\dot{\mathrm{x}}_{\mathrm{Bi}}=\dot{\mathrm{x}}-\left(\mathrm{PB}_{\mathrm{ix}}^{\prime} \sin \phi+\mathrm{PB}_{\mathrm{iy}}^{\prime} \cos \phi\right) \dot{\phi} \\
\dot{\mathrm{y}}_{\mathrm{Bi}}=\dot{\mathrm{y}}+\left(\mathrm{PB}_{\mathrm{ix}}^{\prime} \cos \phi-\mathrm{PB}_{\mathrm{iy}}^{\prime} \sin \phi\right) \dot{\phi}
\end{gathered}
$$

Substituting Eqs.(8) in Eq.(7) and dividing both sides by $\mathrm{d}_{\mathrm{i} 2}$ we get:

$$
\begin{aligned}
\dot{\mathrm{d}}_{\mathrm{i} 2} & =\left(\dot{\mathrm{x}}-\left(\mathrm{PB}_{\mathrm{ix}}^{\prime} \sin \phi+\mathrm{PB}_{\mathrm{iy}}^{\prime} \cos \phi\right) \dot{\phi}-\dot{\mathrm{d}}_{\mathrm{i} 1} \hat{\mathrm{n}}_{\mathrm{i} 1 \mathrm{x}}\right) \hat{\mathrm{n}}_{\mathrm{i} 2 \mathrm{x}} \\
& +\left(\dot{\mathrm{y}}+\left(\mathrm{PB}_{\mathrm{ix}}^{\prime} \cos \phi-P \mathrm{~B}_{\mathrm{iy}}^{\prime} \sin \phi\right) \dot{\phi}-\dot{\mathrm{d}}_{\mathrm{i} 1} \hat{\mathrm{n}}_{\mathrm{i} 1 \mathrm{y}}\right) \hat{\mathrm{n}}_{\mathrm{i} 2 \mathrm{y}}
\end{aligned}
$$

Where, $\hat{\mathrm{n}}_{\mathrm{ilx}}$ and $\hat{\mathrm{n}}_{\mathrm{ily}}$ are the unit vectors of first prismatic joint along global $\mathrm{X}$ and $\mathrm{Y}$ directions corresponding to the limb $i$.

Similarly, $\quad \hat{n}_{\mathrm{i} 2 \mathrm{x}}=\frac{\left(\mathrm{x}_{\mathrm{Bi}}-\mathrm{x}_{\mathrm{Ai}}\right)}{\mathrm{d}_{\mathrm{i} 2}}$ and $\hat{\mathrm{n}}_{\mathrm{i} 2 \mathrm{y}}=\frac{\left(\mathrm{y}_{\mathrm{Bi}}-\mathrm{y}_{\mathrm{Ai}}\right)}{\mathrm{d}_{\mathrm{i} 2}}$ are the components of unit vectors for distal prismatic joints along $A_{i}$ to $B_{i}$. Equation (9) gives the velocity of distal prismatic joints in terms of Cartesian velocities $(\dot{x}, \dot{y}, \dot{\phi})$ and velocities of the base prismatic joints $\dot{\mathrm{d}}_{\mathrm{i} 1}$. In Eq. (9), if all $\dot{\mathrm{d}}_{\mathrm{i} 1}=0$, then it reduces to standard 3-RPR parallel manipulator Jacobian matrix [J] which can be expressed as: $\{\dot{\mathrm{d}}\}=[\mathrm{J}]^{-1}\{\dot{\mathrm{X}}\}$. Importance of Jacobian matrix can also be found in estimating the singular configurations in the workspace.

\subsection{Joint Torques}

Kinetostatics refers to the situation where the actuators move at a constant speed and it does not cause the inertia forces to vanish, since non-zero centripetal and Coriolis force arise essentially out of accelerations of the links even when the actuators do not accelerate. The most common applications of such analysis is found in mechanism for machine tools, micropositioning, surgical robots etc. In this approach, the right side term of Newton-Euler equations reduces to zero. Therefore, there is no dependency on velocities and accelerations and the entire workspace of a certain working mode can be analyzed.
The vector of distal joint torques $\left\{\tau_{2}\right\}$ are obtained in terms of the output wrench (forces at the mobile platform center) $\{\mathrm{F}\}$ as $\left\{\tau_{2}\right\}=[\mathrm{J}]\{\mathrm{F}\}$. However, for base prismatic joints, the force vector from geometry and vector mechanics is given as follows: $\left\{\tau_{1}\right\}=\hat{\mathrm{n}}_{\mathrm{i} 2} \hat{\mathrm{n}}_{\mathrm{i} 1}\left\{\tau_{2}\right\}$, where $\hat{\mathrm{n}}_{\mathrm{i} 1}$ is a unit vector from $\mathrm{O}_{\mathrm{i}}$ to $\mathrm{A}_{\mathrm{i}}$.

\section{RESULTS AND DISCUSSION}

Let $\ell$ is side of mobile platform and $r=\ell / \sqrt{3}$ is equivalent radius of inscribed circle; $\mathrm{t}_{1}=\mathrm{x}_{\mathrm{O} 2}, \mathrm{t}_{2}=\mathrm{x}_{\mathrm{O} 3}, \mathrm{t}_{3}=\mathrm{y}_{\mathrm{O} 3}$ are the coordinates of the base triangle with respect to inertial frame of reference. Taking first time derivative on both sides and simplifying, we get:

$$
\begin{gathered}
\left\{\dot{\mathrm{x}}+\mathrm{r} \dot{\phi} \sin (\phi+\pi / 6)-\dot{\mathrm{d}}_{11}\right\} \hat{\mathrm{n}}_{12 \mathrm{x}} \\
+\{\dot{\mathrm{y}}-\mathrm{r} \dot{\phi} \cos (\phi+\pi / 6)\} \hat{\mathrm{n}}_{12 \mathrm{y}}=\dot{\mathrm{d}}_{12} \\
\left(\dot{\mathrm{x}}+\mathrm{r} \dot{\phi} \sin (\phi+\pi / 6)-\ell \dot{\phi} \sin \phi+\dot{\mathrm{d}}_{21} \cos (\pi / 3)\right) \hat{\mathrm{n}}_{22 \mathrm{x}} \\
+\left(\dot{\mathrm{y}}-\mathrm{r} \dot{\phi} \cos (\phi+\pi / 6)+\ell \dot{\phi} \cos \phi-\dot{\mathrm{d}}_{21} \sin (\pi / 3)\right) \hat{\mathrm{n}}_{22 \mathrm{y}}=\dot{\mathrm{d}}_{22} \\
\left(\dot{\mathrm{x}}+\mathrm{r} \dot{\phi} \sin (\phi+\pi / 6)-\ell \dot{\phi} \sin (\phi+\pi / 3)+\dot{\mathrm{d}}_{31} \cos (\pi / 3)\right) \hat{\mathrm{n}}_{32 \mathrm{x}} \\
+(\dot{\mathrm{y}}-\mathrm{r} \dot{\phi} \cos (\phi+\pi / 6)+\ell \dot{\phi} \cos (\phi+\pi / 3) \\
\left.+\dot{\mathrm{d}}_{31} \sin (\pi / 3)\right) \hat{\mathrm{n}}_{32 \mathrm{y}}=\dot{\mathrm{d}}_{32}
\end{gathered}
$$

It is noticed that $\hat{\mathrm{n}}_{\mathrm{i} 1 \mathrm{x}}$ and $\hat{\mathrm{n}}_{\mathrm{ily}}$ in Eq.(9) are unit vector components for each link-orientations derived as follows:

$$
\begin{aligned}
& \hat{\mathrm{n}}_{12 \mathrm{x}}=\left\{\mathrm{x}-\mathrm{r} \cos (\phi+\pi / 6)-\mathrm{d}_{11}\right\} / \mathrm{d}_{12} \\
& \hat{\mathrm{n}}_{12 \mathrm{y}}=\{\mathrm{y}-\mathrm{r} \sin (\phi+\pi / 6)\} / \mathrm{d}_{12} \\
& \hat{\mathrm{n}}_{22 \mathrm{x}}=\left\{\mathrm{x}-\mathrm{r} \cos (\phi+\pi / 6)+\ell \cos \phi-\left(\mathrm{t}_{1}-\mathrm{d}_{21} \cos (\pi / 3)\right\} / \mathrm{d}_{22}\right. \\
& \hat{\mathrm{n}}_{22 \mathrm{y}}=\left\{\mathrm{y}-\mathrm{r} \sin (\phi+\pi / 6)+\ell \sin \phi-\mathrm{d}_{21} \sin (\pi / 3)\right\} / \mathrm{d}_{22} \\
& \hat{\mathrm{n}}_{32 \mathrm{x}}= \\
& \frac{1}{\mathrm{~d}_{32}}\left\{\mathrm{x}-\mathrm{r} \cos (\phi+\pi / 6)+\ell \cos (\phi+\pi / 3)-\left(\mathrm{t}_{2}-\mathrm{d}_{31} \cos (\pi / 3)\right\}\right. \\
& \hat{\mathrm{n}}_{32 \mathrm{y}}= \\
& \left\{\mathrm{y}-\mathrm{r} \sin (\phi+\pi / 6)+\ell \sin (\phi+\pi / 3)-\left(\mathrm{t}_{3}-\mathrm{d}_{31} \sin (\pi / 3)\right\} / \mathrm{d}_{32}\right.
\end{aligned}
$$

The dimensions chosen for the manipulator are $\mathrm{O}_{1} \mathrm{O}_{2}=\mathrm{O}_{2} \mathrm{O}_{3}=$ $\mathrm{O}_{3} \mathrm{O}_{1}=0.3 \mathrm{~m}$ and $\mathrm{B}_{1} \mathrm{~B}_{2}=\mathrm{B}_{2} \mathrm{~B}_{3}=\mathrm{B}_{3} \mathrm{~B}_{1}=0.05 \mathrm{~m}$. Computer programs are developed in MATLAB for workspace analysis, Jacobain and force analysis. Following input data is 
considered: The range limits on these prismatic joints are set to $0.01 \mathrm{~m}$ to $0.29 \mathrm{~m}$. Also $\phi \in\left[-180^{\circ}, 180^{\circ}\right]$. The workspace of non-redundant 3-RPR linkage is shown in Fig.2. It is generated from inverse kinematics and by a method called direct search approach. Here initially, we vary $\mathrm{x}, \mathrm{y}$ and $\phi$ over a range (in present case $\mathrm{x}, \mathrm{y} \in[-0.4,0.4]$ ) and predict $\mathrm{d}_{2 \mathrm{i}}$ values; if $\left(\mathrm{d}_{2 \mathrm{i}}\right)_{\min }<\mathrm{d}_{2 \mathrm{i}}<\left(\mathrm{d}_{2 \mathrm{i}}\right)_{\max }$, the selected point falls into the reachable workspace at that orientation.

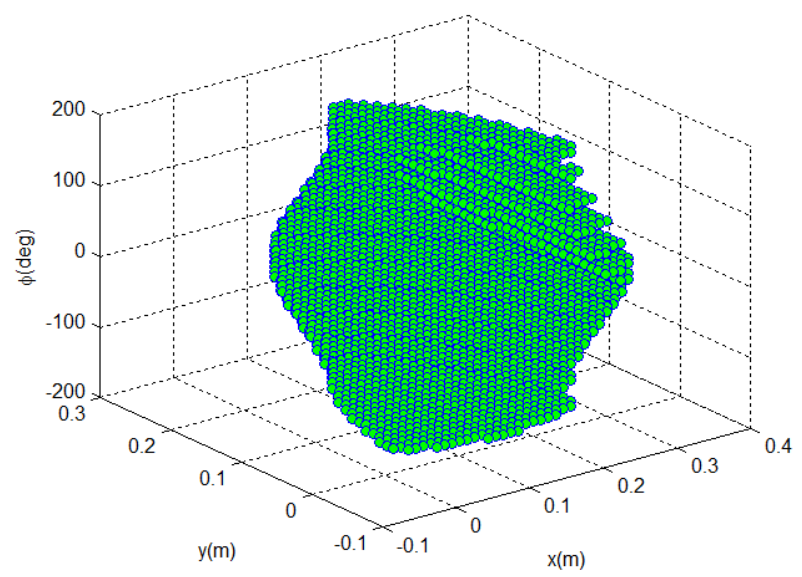

Fig.2 Three-dimensional workspace of 3-RPR linkage

The workspace of redundant 3-PRPR manipulator for an arbitrary values of $\mathrm{d}_{11}=0.1613 \mathrm{~m}, \mathrm{~d}_{21}=0.2900 \mathrm{~m}$ and $\mathrm{d}_{31}=0.1212 \mathrm{~m}$ is shown in Fig.3. It is seen that an increased workspace is obtained due to additional prismatic joints in all the orientations of the platform.

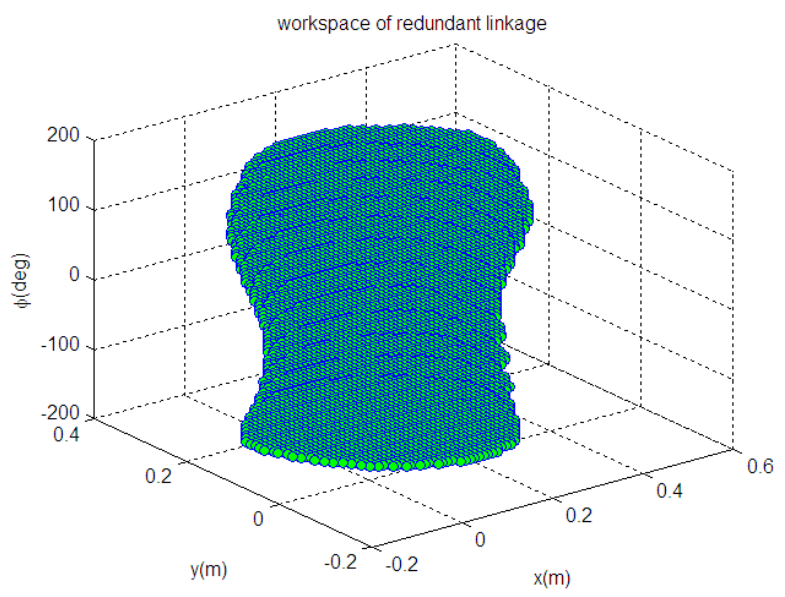

Fig.3 Workspace of 3-PRRR in constant arbitrary base posture

The velocity kinematics of manipulators is next studied with a trajectory involving a straight-line path within the constant- orientation workspace as shown in Fig.4. It may or may not pass through the singular points.

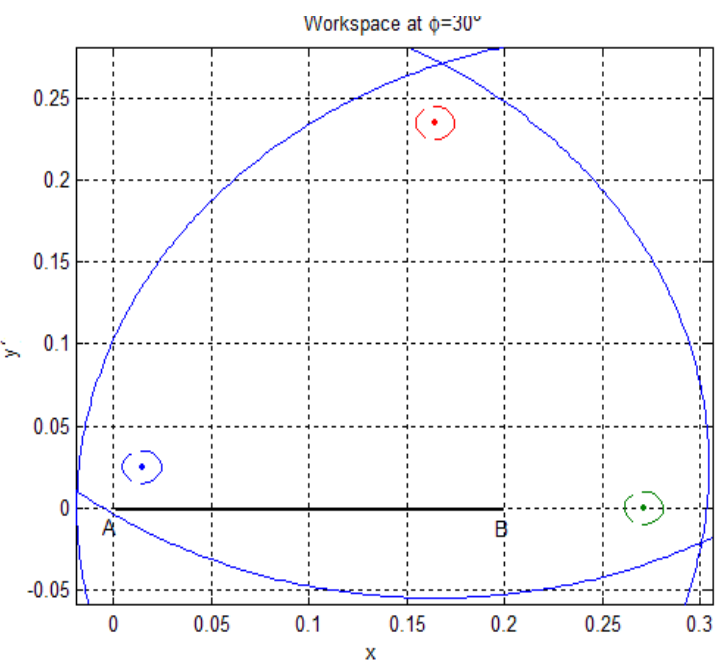

Fig.4 Horizontal line trajectory within the workspace envelope

The platform is moving from the center $\mathrm{A}(0,0)$ to $\mathrm{B}(0.2,0)$ in increments of $0.004 \mathrm{~m}$. A constant force of $100 \mathrm{~N}$ is applied on the platform in the direction opposite to the motion; thus the force vector at the platform is $[-100 ; 0 ; 0]$ N. Fig. 5 shows the displacements at the joints during each time step as obtained from Jacobian analysis.

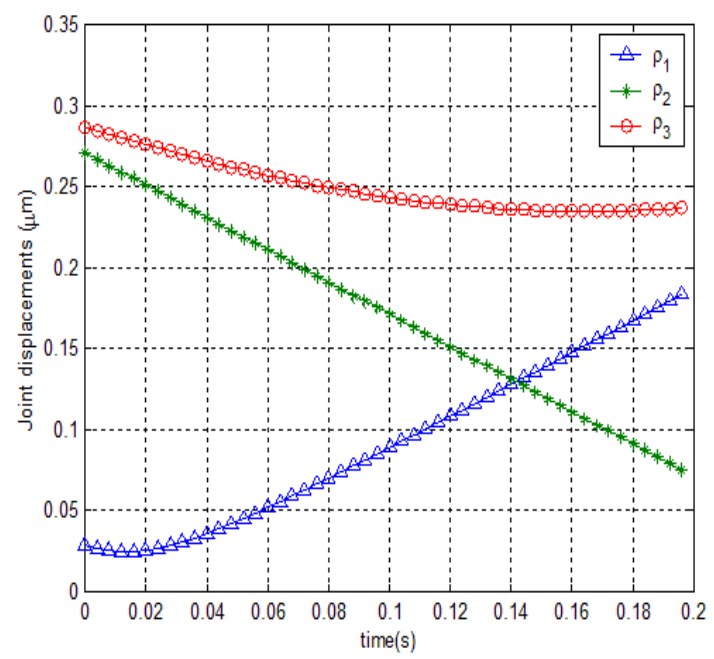

Fig.5 Joint displacements corresponding to straight line track

The corresponding generalized forces required for the nonredundant manipulator are shown in Fig.6. 


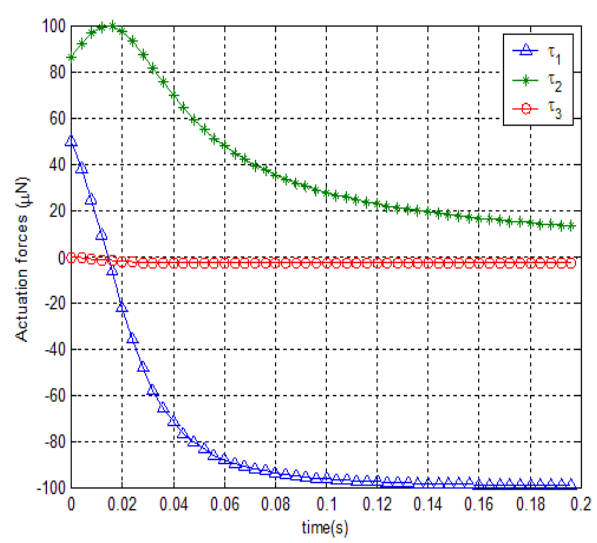

Fig.6 Joint forces for non-redundant configuration

It can be seen that the maximum forces occur at the starting point of the trajectory, where it is touching the boundary of workspace. This is a point of force (type-II) singularity. It is assumed that the mobile platform is moving with constant velocity in Cartesian plane. Further by adding additional prismatic joints with the initial joint displacements: $0.16 \mathrm{~m}$, $0.29 \mathrm{~m}$ and $0.12 \mathrm{~m}$, the higher joint forces are not acting for long duration as seen in Fig.7 for the same straight line trajectory.

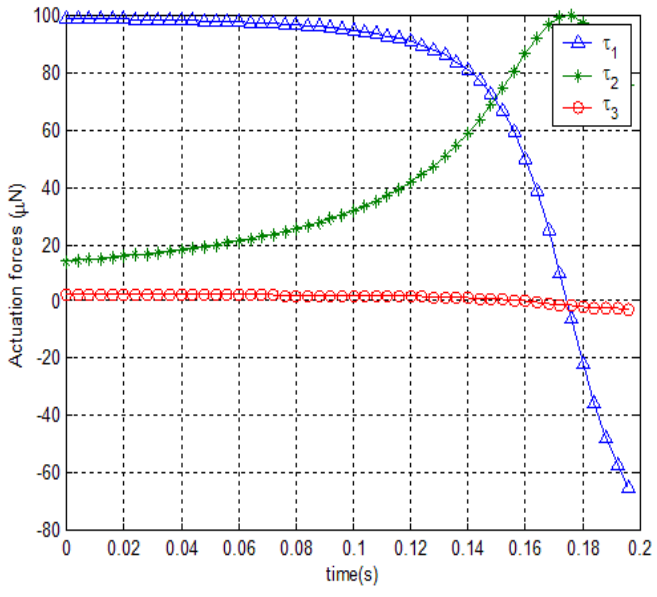

Fig.7. Joint actuation forces for redundant counterpart

Based on the advantages of this kinematically redundant linkage, a prototype is planned to be fabricated. Here it is assumed that the three actuated sliding joints are provided with the electrostatic actuation using comb drives. The pivot points $(\mathrm{R})$ in the design are to be replaced by flexure hinges [11] which allow in-plane rotation and the controlled prismatic joints are to be implemented by linear comb actuators with folded suspension springs to restore the joint to its original position where the comb drives are de-energized. To incorporate the effects of flexure hinge stiffness on the joint motions, the resultant equations are to be slightly modified.
Fig. 8 shows the screen shot of the table with one of leg of this monolithic compliant parallel linkage developed in ANSYS environment. The comb-drives will be provided beneath the middle dyad link and platform as well as base slider is respectively connected to this dyad using flexure joints. Base prismatic joint is also actuated with electro-static actuation with the help of a capacitor plate.

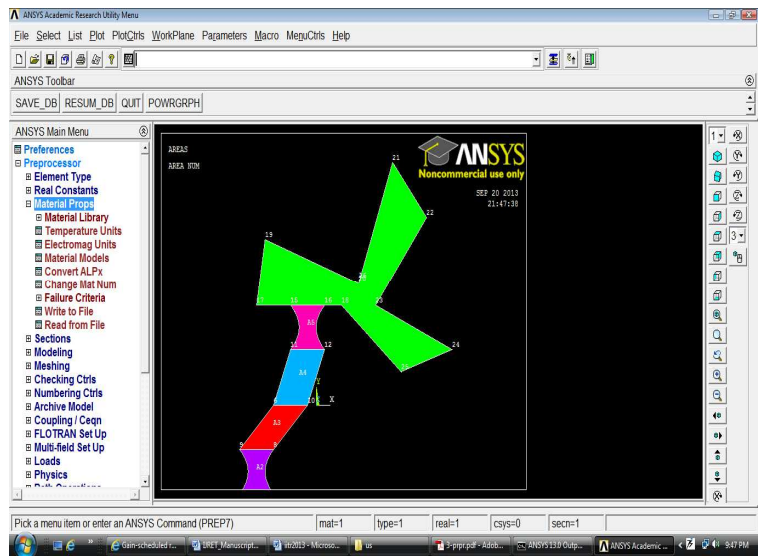

Fig.8 Surface model of the proposed positioning stage

The static analysis provides the approximate Jacobian matrix in one of the postures of the linkage. Modal analysis gives an estimation of natural frequencies of the stage.

\section{CONCLUSIONS}

A kinematically redundant planar parallel manipulator was considered in this paper. Inverse kinematics and velocity analysis of the linkage were mathematically explained. The reachable workspaces were obtained and compared to that of similar-sized non-redundant manipulator. It was shown that the proposed manipulator's workspaces were significantly larger at all platform orientations. For a straight line trajectory, the corresponding joint actuation forces have been computed and it was shown that the mobile platform reaches the points with little joint forces. In force-analysis, the arbitrarily locations of base prismatic joints were initially selected. However, in practical case it may be casted as an optimum design problem where these locations are selected for either maximizing stiffness or minimizing joint torques at each and every point along the trajectory. Since, the manipulator is kinematically redundant for every point inside the reachable workspace, there are loci of solutions of the inverse displacement problem for a given end effector pose. Therefore, it is possible to choose sets of fixed solutions that are singular-free.

\section{ACKNOWLEDGEMENTS}

The authors acknowledge Sri Chandra Sekharendra Viswa Mahavidyalaya, Kancheepuram. 


\section{REFERENCES}

[1]. D. Stewart. 'A platform with six degrees of freedom', Proc Inst Mech Eng., vol.180 (1), pp. 371-386, 1965.

[2]. Bonev, 'Delta parallel robot', http://www.parallemic.org/Reviews/Review002.html.

[3]. B.S. El-Khasawneh, P.M. Ferreira, C.R. Boër, L. Molinari-Tosatti and K.S. Smith (Eds.), 'The tetrahedral tripod, parallel-kinematic machinestheoretical aspects and industrial requirements', Springer-Verlag pp. 419-430, 1999.

[4]. J. Wang and C.M. Gosselin, 'Kinematic analysis and design of kinematically redundant parallel mechanisms', ASME Journal of Mechanical Design, vol.126 (1), pp.109-118, 2004.

[5]. M.G. Mohamed and C.M. Gosselin, 'Design and analysis of kinematically redundant parallel manipulators with configurable platforms', IEEE Transactions on Robotics, vol.21 (3), pp.277-287, 2005.

[6]. I. Ebrahimi, J.A. Carretero and R. Boudreau, '3-PRRR redundant planar parallel manipulator: inverse displacement, workspace and singularity analyses', Mechanism and Machine Theory, vol. 42 (8), pp.10071016, 2007.

[7]. J. Kotlarski, T.D. Thanh, B. Heimann and T. Ortmaier, 'Optimization strategies for additional actuators of kinematically redundant parallel kinematic machines', Proceedings of IEEE Conference on Robotics and Automation, pp. 656-661, 2010.

[8]. R. Boudreau and S.Nokleby, 'Force optimization of kinematically-redundant planar parallel manipulators following a desired trajectory', Mechanisms and Machine Theory, vol.56, pp.138-155, 2012.

[9]. C.Shn, T.Seo, J.Kim and T.W.Kim, 'High-tilt parallel positioning mechanism development and cutter path simulation for laser micro-machining', ComputerAided Design, vol.39, pp.218-228, 2007.

[10]. S.Krishnan and L.Saggere, 'Design and Development of a novel micro-clasp gripper for micromanipulation of complex shaped objects', Sensors and Actuators, A:Physical, vol.176, pp.110-123, 2012.

[11]. D.Mukhopadhyay, J.Dong, E.Pengwang and P.Ferreira, 'A SOI-MEMS-based 3-DOF planar parallel kinematics nanopositioning stage', Sensors and Actuators: A, vol.147, pp.340-351, 2008.

\section{BIOGRAPHIES}

Er .U. Sudhakar is working as Assistant Professor at GMRITRajam. To his credit he published around 5 articles in national conferences. His fields of interest include: manufacturing techniques, parallel robots and MEMS.

Dr. J. Srinivas, a member of Institution of Engineers (India) has around 15 years of research and teaching experience. He has published around 70 research articles in various conferences and journals. He authored 8 textbooks relating to Mechanical Engineering. His topics of interest are dynamics and control, micro positioning analysis and MEMS and NEMS. 